\title{
Linear coupling parametrization in the action-angle frame
}

\author{
Yun Luo* \\ Brookhaven National Laboratory, Upton, New York 11973, USA
}

(Received 2 September 2004; published 7 December 2004)

\begin{abstract}
The linear coupling's parameterization in the uncoupled action-angle frame is introduced, where the two eigenmodes' phases at one point and the phase advances between two points in the ring are well defined. The general expressions of the coordinates $\left(x, x^{\prime}, y, y^{\prime}\right)$ are also given. The transfer matrix $\mathbf{P}$ from the action-angle frame to the laboratory frame can be obtained from the eigenvectors of the one-turn transfer map $\mathbf{T}$ or from Twiss and coupling parameters defined in Edwards-Teng parameterization. Matrix $\mathbf{P}$ can also be constructed from turn-by-turn beam position monitor (BPM) data. The phase ellipses and beam sizes in different projection planes are calculated through the action-angle parameterization. The two eigenmodes' Twiss and coupling parameters at point $s_{2}$ are described in terms of the section transfer map $\mathbf{T}_{1 \rightarrow 2}$ and parameters at point $s_{1}$. The linear coupling's action-angle parameterization is useful for the analytical calculations and turn-by-turn BPM data interpretations.
\end{abstract}

DOI: 10.1103/PhysRevSTAB.7.124001

PACS numbers: 29.20.Dh, 29.27.Bd

\section{INTRODUCTION}

The Courant-Snyder parameterization [1] of a particle's uncoupled one-dimensional betatron oscillation in circular accelerators is a great success. Work continues to describe coupled two-dimensional transverse betatron motion in an elegant way as the one-dimensional parameterization. Here another approach is demonstrated.

There are two main methods to parametrize twodimensional linearly coupled betatron motion. One is to start from eigenvectors of the one-turn $4 \times 4$ transfer matrix $\mathbf{T}$ to construct the particle's four coordinates $\left(x, x^{\prime}, y, y^{\prime}\right)$ in the laboratory frame. The coordinates in the eigenvector frame keep constant while the eigenvectors transfer from one point to another in the ring. Comparing to the one-dimensional Courant-Snyder parametrization, new sets of Twiss parameters in the two-dimensional situation are defined accordingly. One well-known parametrization in this direction was published by Ripken [2-4]. There, four $\beta$ and four $\alpha$ functions are defined. Another approach in this direction has been reported recently by Lebedev and Bogacz [5].

Another approach is to directly decouple the one-turn transfer map into an uncoupled one-turn map through a matrix similarity transformation. One successful approach in this direction was obtained by Edwards and Teng [6,7] and improved by others [8-11]. There, two sets of Twiss parameters for the two eigenmodes and a coupling matrix $\mathbf{C}$ are introduced. This parametrization has been widely used in coupling measurements, where a normalized $\overline{\mathbf{C}}$ is used [10,12].

Ripken's parametrization gives straightforward expressions of the particle's coordinates in the laboratory frame. However, this parametrization is complicated. EdwardsTeng parametrization is based on matrix manipulations.

*Electronic address: yluo@bnl.gov
The transformations between the one-turn map and its defined Twiss and coupling parameters can be easily obtained. A problem of this parametrization is its ambiguous definitions of betatron phases and phase advances. The relation between the one-turn transfer map's eigenvectors and its defined Twiss and coupling parameters is not given.

With high performance digital beam position monitor (BPM) and modern analytical techniques [13,14], phases and phase advances for the two eigenmodes can be measured very precisely. The merit of the phase measurement is that it is insensitive to BPM offsets and gains. For a given betatron oscillation, the two eigenmodes' phases and phase advances are all well defined. So it is natural to include them in a linear coupling's parametrization.

In this article we first introduce the action-angle frame, then we demonstrate the methods to construct matrix $\mathbf{P}$ both analytically and experimentally. The phase ellipses, their areas and beam sizes are calculated through the action-angle parametrization. $\mathbf{P}$ and Twiss parameters at point $s_{2}$ are obtained in terms of the section transfer matrix $\mathbf{T}_{1 \rightarrow 2}$ and matrix $\mathbf{P}$ at point $s_{1}$.

\section{ACTION-ANGLE FRAME}

For general two-dimensional linearly coupled motion, single-particle motion can be written as

$$
\left(\begin{array}{c}
x \\
x^{\prime} \\
y \\
y^{\prime}
\end{array}\right)=\mathbf{P}\left(\begin{array}{c}
\sqrt{2 J_{I}} \cos \Phi_{I} \\
-\sqrt{2 J_{I}} \sin \Phi_{I} \\
\sqrt{2 J_{I I}} \cos \Phi_{I I} \\
-\sqrt{2 J_{I I}} \sin \Phi_{I I}
\end{array}\right)
$$

where $J_{I, I I}$ are the constant actions of the two eigenmodes and $\Phi_{I, I I}$ are the eigenmode phases. One-turn phase advances for the two eigenmodes are $2 \pi \mu_{I, I I}$. The $4 \times 4$ matrix $\mathbf{P}$ transfers the coordinates from the new frame to the laboratory frame. 
The transfer matrix in the new frame is the rotation matrix $\mathbf{R}\left(\Delta \Phi_{I}, \Delta \Phi_{I I}\right)$, where $\Delta \Phi_{I}, \Delta \Phi_{I I}$ are the phase advances between two points in the ring for the two eigenmodes, respectively. The rotation matrix is

$$
\mathbf{R}\left(\Delta \Phi_{I}, \Delta \Phi_{I I}\right)=\left(\begin{array}{cc}
\mathbf{R}\left(\Delta \Phi_{I}\right) & \mathbf{0} \\
\mathbf{0} & \mathbf{R}\left(\Delta \Phi_{I I}\right)
\end{array}\right),
$$

where

$$
\mathbf{R}\left(\Delta \Phi_{i}\right)=\left(\begin{array}{cc}
\cos \Delta \Phi_{i} & \sin \Delta \Phi_{i} \\
-\sin \Delta \Phi_{i} & \cos \Delta \Phi_{i}
\end{array}\right) .
$$

It is easy to prove that the two eigenmodes are uncoupled in the new frame. The new frame is named as the actionangle frame.

\section{A. Betatron phases}

In Eq. (1), there is still an ambiguity in the definitions of the eigenmode phases $\Phi_{I, I I}$. Here we define $\Phi_{I}$ as the phase of eigenmode I's contribution to the $x$ coordinate, and $\Phi_{I I}$ as the phase of eigenmode II's contribution to the $y$ coordinate. Therefore, the elements $p_{12}$ and $p_{34}$ in $\mathbf{P}$ are both zero,

$$
\mathbf{P}=\left(\begin{array}{cccc}
p_{11} & 0 & p_{13} & p_{14} \\
p_{21} & p_{22} & p_{23} & p_{24} \\
p_{31} & p_{32} & p_{33} & 0 \\
p_{41} & p_{42} & p_{43} & p_{44}
\end{array}\right)
$$

The coordinates in the laboratory frame are given by

$$
\left\{\begin{array}{rl}
x & =p_{11} \sqrt{2 J_{I}} \cos \Phi_{I}+p_{13} \sqrt{2 J_{I I}} \cos \Phi_{I I}-p_{14} \sqrt{2 J_{I I}} \sin \Phi_{I I} \\
x^{\prime} & =p_{21} \sqrt{2 J_{I}} \cos \Phi_{I}-p_{22} \sqrt{2 J_{I}} \sin \Phi_{I}+p_{23} \sqrt{2 J_{I I}} \cos \Phi_{I I}-p_{24} \sqrt{2 J_{I I}} \sin \Phi_{I I} \\
y & =p_{31} \sqrt{2 J_{I}} \cos \Phi_{I}-p_{32} \sqrt{2 J_{I}} \sin \Phi_{I}+p_{33} \sqrt{2 J_{I I}} \cos \Phi_{I I} \\
y^{\prime} & =p_{41} \sqrt{2 J_{I}} \cos \Phi_{I}-p_{42} \sqrt{2 J_{I}} \sin \Phi_{I}+p_{43} \sqrt{2 J_{I I}} \sin \Phi_{I I}-p_{44} \sqrt{2 J_{I I}} \sin \Phi_{I I}
\end{array} .\right.
$$

Equation (5) is the general expression for $\left(x, x^{\prime}, y, y^{\prime}\right)$ coordinates of single-particle linear motion. It has very clear physical meanings, and therefore is convenient for the experimental turn-by-turn BPM data interpretations and analytical calculations.

In the uncoupled case, all elements in the off-diagonal blocks of matrix $\mathbf{P}$ are zero. The relationship of $p_{i j}$ 's and Twiss parameters defined in Courant-Snyder parametrization can be easily found.

\section{B. One eigenmode excitation}

To measure the betatron optics, a single eigenmode motion is coherently excited $[15,16]$. If only eigenmode I is excited, the coordinates of the bunch center are given by

$$
\left(\begin{array}{c}
x \\
x^{\prime} \\
y \\
y^{\prime}
\end{array}\right)=\mathbf{P}\left(\begin{array}{c}
\sqrt{2 J_{I}} \cos \Phi_{I} \\
-\sqrt{2 J_{I}} \sin \Phi_{I} \\
0 \\
0
\end{array}\right)
$$

or

$$
\left\{\begin{array}{l}
x=p_{11} \sqrt{2 J_{I}} \cos \Phi_{I} \\
x^{\prime}=p_{21} \sqrt{2 J_{I}} \cos \Phi_{I}-p_{22} \sqrt{2 J_{I}} \sin \Phi_{I} \\
y=p_{31} \sqrt{2 J_{I}} \cos \Phi_{I}-p_{32} \sqrt{2 J_{I}} \sin \Phi_{I} \\
y^{\prime}=p_{41} \sqrt{2 J_{I}} \cos \Phi_{I}-p_{42} \sqrt{2 J_{I}} \sin \Phi_{I}
\end{array} .\right.
$$

If only eigenmode II is excited, the coordinates of the bunch center are given by

$$
\left(\begin{array}{c}
x \\
x^{\prime} \\
y \\
y^{\prime}
\end{array}\right)=\mathbf{P}\left(\begin{array}{c}
0 \\
0 \\
\sqrt{2 J_{I I}} \cos \Phi_{I I} \\
-\sqrt{2 J_{I I}} \sin \Phi_{I I}
\end{array}\right)
$$

or

$$
\left\{\begin{array}{rl}
x & =p_{13} \sqrt{2 J_{I I}} \cos \Phi_{I I}-p_{14} \sqrt{2 J_{I I}} \sin \Phi_{I I} \\
x^{\prime} & =p_{23} \sqrt{2 J_{I I}} \cos \Phi_{I I}-p_{24} \sqrt{2 J_{I I}} \sin \Phi_{I I} \\
y & =p_{33} \sqrt{2 J_{I I}} \cos \Phi_{I I} \\
y^{\prime} & =p_{43} \sqrt{2 J_{I I}} \cos \Phi_{I I}-p_{44} \sqrt{2 J_{I I}} \sin \Phi_{I I}
\end{array} .\right.
$$

\section{Matrix $P$ and section transfer matrix $T_{1 \rightarrow 2}$}

The coordinates in the laboratory frame at point $s_{2}$ are obtained from those at point $s_{1}$ by

$$
\left(\begin{array}{c}
x \\
x^{\prime} \\
y \\
y^{\prime}
\end{array}\right)_{2}=\mathbf{T}_{1 \rightarrow 2}\left(\begin{array}{c}
x \\
x^{\prime} \\
y \\
y^{\prime}
\end{array}\right)_{1}
$$

or through the action-angle frame

$$
\left(\begin{array}{c}
x \\
x^{\prime} \\
y \\
y^{\prime}
\end{array}\right)_{2}=\mathbf{P}_{2} \mathbf{R}\left(\Delta \Phi_{I}, \Delta \Phi_{I I}\right) \mathbf{P}_{1}^{-1}\left(\begin{array}{c}
x \\
x^{\prime} \\
y \\
y^{\prime}
\end{array}\right)_{1} .
$$

So one obtains

$$
\mathbf{T}_{1 \rightarrow 2}=\mathbf{P}_{2} \mathbf{R}\left(\Delta \Phi_{I}, \Delta \Phi_{I I}\right) \mathbf{P}_{1}^{-1} .
$$

If the phase advances between the two points are known, $\mathbf{P}_{2}$ at point $s_{2}$ can be obtained with the section transfer map $\mathbf{T}_{1 \rightarrow 2}$ and matrix $\mathbf{P}_{1}$,

$$
\mathbf{P}_{2}=\mathbf{T}_{1 \rightarrow 2} \mathbf{P}_{1} \mathbf{R}^{-1}\left(\Delta \Phi_{I}, \Delta \Phi_{I I}\right) .
$$

The one-turn transfer map at one point is

$$
\mathbf{T}=\mathbf{P R}\left(2 \pi \mu_{I}, 2 \pi \mu_{I I}\right) \mathbf{P}^{-1} .
$$




\section{CONSTRUCTION OF MATRIX P}

\section{A. Matrix $P$ and eigenvectors}

The four eigenvectors of the one-turn transfer matrix $\mathbf{T}$ at one point are $\mathbf{v}_{1}, \mathbf{v}_{1}^{*}, \mathbf{v}_{2}, \mathbf{v}_{2}^{*}$,

$$
\left\{\begin{array}{l}
\mathbf{T} \mathbf{v}_{1}=\lambda_{1} \mathbf{v}_{1}=e^{i 2 \pi \mu_{I}} \mathbf{v}_{1} \\
\mathbf{T} \mathbf{v}_{1}^{*}=\lambda_{2} \mathbf{v}_{1}^{*}=e^{-i 2 \pi \mu_{I}} \mathbf{v}_{1}^{*} \\
\mathbf{T}_{2}=\lambda_{3} \mathbf{v}_{2}=e^{i 2 \pi \mu_{I I}} \mathbf{v}_{2} \\
\mathbf{T}_{2}^{*}=\lambda_{4} \mathbf{v}_{2}^{*}=e^{-i 2 \pi \mu_{I I}} \mathbf{v}_{2}^{*}
\end{array} .\right.
$$

Substituting Eq. (14),

$$
\left\{\begin{array}{l}
\mathbf{R}\left(2 \pi \mu_{I}, 2 \pi \mu_{I I}\right)\left(\mathbf{P}^{-} \mathbf{v}_{1}\right)=e^{i 2 \pi \mu_{I}\left(\mathbf{P}^{-} \mathbf{v}_{1}\right)} \\
\mathbf{R}\left(2 \pi \mu_{I}, 2 \pi \mu_{I I}\right)\left(\mathbf{P}^{-} \mathbf{v}_{1}^{*}\right)=e^{-i 2 \pi \mu_{I}\left(\mathbf{P}^{-} \mathbf{v}_{1}^{*}\right)} \\
\mathbf{R}\left(2 \pi \mu_{I}, 2 \pi \mu_{I I}\right)\left(\mathbf{P}^{-} \mathbf{v}_{2}\right)=e^{i 2 \pi \mu_{I I}\left(\mathbf{P}^{-} \mathbf{v}_{2}\right)} \\
\mathbf{R}\left(2 \pi \mu_{I}, 2 \pi \mu_{I I}\right)\left(\mathbf{P}^{-} \mathbf{v}_{2}^{*}\right)=e^{-i 2 \pi \mu_{I I}\left(\mathbf{P}^{-} \mathbf{v}_{2}^{*}\right)}
\end{array}\right.
$$

The eigenvectors of the one-turn transfer map are normalized according to

$$
\mathbf{v}_{i}^{T} \mathbf{v}_{i}^{*}=1
$$

However, after the normalization, there are still phase uncertainties. To assure that $p_{12}$ and $p_{34}$ are zero, the following procedures are needed. If the phase of the first element of $\mathbf{v}_{1}$ is $\theta_{I}$, one multiplies $\mathbf{v}_{1}$ by $e^{i\left(-\pi / 2-\theta_{I}\right)}$. Then the phase of the first element of $\mathbf{v}_{1}^{*}$ is $-\theta_{I}$, one multiplies $\mathbf{v}_{1}^{*}$ by $e^{i\left(\pi / 2+\theta_{I}\right)}$. If the phase of the third element of $\mathbf{v}_{2}$ is $\theta_{I I}$, one multiplies $\mathbf{v}_{2}$ by $e^{i\left(-\pi / 2-\theta_{I I}\right)}$. Then the phase of the third element of $\mathbf{v}_{2}^{*}$ is $-\theta_{I I}$, one multiplies $\mathbf{v}_{2}^{*}$ by $e^{i\left(\pi / 2+\theta_{I I}\right)}$.

The rotation matrix $\mathbf{R}\left(2 \pi \mu_{I}, 2 \pi \mu_{I I}\right)$ has four normalized eigenvectors $\tilde{\mathbf{v}}_{1}, \tilde{\mathbf{v}}_{1}^{*}, \tilde{\mathbf{v}}_{2}, \tilde{\mathbf{v}}_{2}^{*}$. And it has the same eigenvalues as these of one-turn transfer matrix $\mathbf{T}$.

$$
\begin{aligned}
& \left\{\begin{array}{l}
\mathbf{R}\left(2 \pi \mu_{I}, 2 \pi \mu_{I I}\right) \tilde{\mathbf{v}}_{1}=\lambda_{1} \tilde{\mathbf{v}}_{1}=e^{i 2 \pi \mu_{I}} \tilde{\mathbf{v}}_{1} \\
\mathbf{R}\left(2 \pi \mu_{I}, 2 \pi \mu_{I I}\right) \tilde{\mathbf{v}}_{1}^{*}=\lambda_{2} \tilde{\mathbf{v}}_{1}^{*}=e^{-i 2 \pi \mu_{I} \tilde{\mathbf{v}}_{1}^{*}} \\
\mathbf{R}\left(2 \pi \mu_{I}, 2 \pi \mu_{I I}\right) \tilde{\mathbf{v}}_{2}=\lambda_{3} \tilde{\mathbf{v}}_{2}=e^{i 2 \pi \mu_{I I} \tilde{\mathbf{v}}_{2}} \\
\mathbf{R}\left(2 \pi \mu_{I}, 2 \pi \mu_{I I}\right) \tilde{\mathbf{v}}_{2}^{*}=\lambda_{4} \tilde{\mathbf{v}}_{2}^{*}=e^{-i 2 \pi \mu_{I I} \tilde{\mathbf{v}}_{2}^{*}}
\end{array},\right. \\
& \left\{\begin{array}{l}
\tilde{\mathbf{v}}_{1}=\frac{1}{\sqrt{2}}\left(\begin{array}{llll}
-\mathrm{i} & 1 & 0 & 0
\end{array}\right)^{T} \\
\tilde{\mathbf{v}}_{1}^{*}=\frac{1}{\sqrt{2}}\left(\begin{array}{llll}
\mathrm{i} & 1 & 0 & 0
\end{array}\right)^{T} \\
\tilde{\mathbf{v}}_{2}=\frac{1}{\sqrt{2}}\left(\begin{array}{llll}
0 & 0 & -\mathrm{i} & 1
\end{array}\right)^{T} \\
\tilde{\mathbf{v}}_{2}^{*}=\frac{1}{\sqrt{2}}\left(\begin{array}{llll}
0 & 0 & \mathrm{i} & 1
\end{array}\right)^{T}
\end{array} .\right.
\end{aligned}
$$

Combining Eqs. (16), (18), and (19), one obtains

$$
\left\{\begin{array}{l}
\mathbf{P}^{-1} \mathbf{v}_{1}=\frac{1}{\sqrt{2}}\left(\begin{array}{llll}
-\mathrm{i} & 1 & 0 & 0
\end{array}\right)^{T} \\
\mathbf{P}^{-1} \mathbf{v}_{1}^{*}=\frac{1}{\sqrt{2}}\left(\begin{array}{llll}
\mathrm{i} & 1 & 0 & 0
\end{array}\right)^{T} \\
\mathbf{P}^{-1} \mathbf{v}_{2}=\frac{1}{\sqrt{2}}\left(\begin{array}{llll}
0 & 0 & -\mathrm{i} & 1
\end{array}\right)^{T} \\
\mathbf{P}^{-1} \mathbf{v}_{2}^{*}=\frac{1}{\sqrt{2}}\left(\begin{array}{llll}
0 & 0 & \mathrm{i} & 1
\end{array}\right)
\end{array} .\right.
$$

Defining four new real vectors from the eigenvectors of one-turn transfer matrix $\mathbf{T}$,

$$
\left\{\begin{array}{l}
\mathbf{e}_{1}=\frac{i}{\sqrt{2}}\left(\mathbf{v}_{1}-\mathbf{v}_{1}^{*}\right) \\
\mathbf{e}_{2}=\frac{1}{\sqrt{2}}\left(\mathbf{v}_{1}+\mathbf{v}_{1}^{*}\right) \\
\mathbf{e}_{3}=\frac{i}{\sqrt{2}}\left(\mathbf{v}_{2}-\mathbf{v}_{2}^{*}\right) \\
\mathbf{e}_{4}=\frac{1}{\sqrt{2}}\left(\mathbf{v}_{2}+\mathbf{v}_{2}^{*}\right)
\end{array},\right.
$$

then from Eq. (20), one gets

$$
\left\{\begin{array}{l}
\mathbf{P}^{-1} \mathbf{e}_{1}=\left(\begin{array}{llll}
1 & 0 & 0 & 0
\end{array}\right)^{T} \\
\mathbf{P}^{-1} \mathbf{e}_{2}=\left(\begin{array}{llll}
0 & 1 & 0 & 0
\end{array}\right)^{T} \\
\mathbf{P}^{-1} \mathbf{e}_{3}=\left(\begin{array}{llll}
0 & 0 & 1 & 0
\end{array}\right)^{T} \\
\mathbf{P}^{-1} \mathbf{e}_{4}=\left(\begin{array}{llll}
0 & 0 & 0 & 1
\end{array}\right)^{T}
\end{array},\right.
$$

or in a compact way,

$$
\mathbf{P}=\mathbf{e},
$$

where matrix $\mathbf{e}$ is constructed as

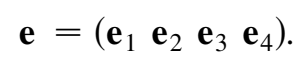

\section{B. Matrix $\mathbf{P}$ and Twiss, coupling parameters}

In Edwards-Teng parametrization, the coupled one-turn transfer matrix is decoupled based on matrix manipulations,

$$
\mathbf{T}=\mathbf{V}\left(\begin{array}{cc}
\mathbf{A} & \mathbf{0} \\
\mathbf{0} & \mathbf{B}
\end{array}\right) \mathbf{V}^{-1}
$$

where

$$
\mathbf{V}=\left(\begin{array}{cc}
r \mathbf{I} & \mathbf{C} \\
-\mathbf{C}^{+} & r \mathbf{I}
\end{array}\right)
$$

In order to easily distinguish Twiss parameter $\gamma$, here we use $r$ in $\mathbf{V}$ instead of $\gamma$ used in other literature. Matrices $\mathbf{A}$ and $\mathbf{B}$ are parametrized like one-dimensional CourantSnyder parametrization [1]. They can be further parametrized as $\mathbf{U}_{i} \mathbf{R}\left(2 \pi \mu_{i}\right) \mathbf{U}_{i}^{-1}$,

$$
\mathbf{U}_{i}=\left(\begin{array}{cc}
\sqrt{\beta_{i}} & 0 \\
\frac{-\alpha_{i}}{\sqrt{\beta_{i}}} & \frac{1}{\sqrt{\beta_{i}}}
\end{array}\right) .
$$

Then one-turn transfer matrix $\mathbf{T}$ is rewritten as

$$
\mathbf{T}=\mathbf{V U R}\left(2 \pi \mu_{I}, 2 \pi \mu_{I I}\right) \mathbf{U}^{-1} \mathbf{V}^{-1},
$$

where

$$
\mathbf{U}=\left(\begin{array}{cc}
\mathbf{U}_{1} & \mathbf{0} \\
\mathbf{0} & \mathbf{U}_{2}
\end{array}\right)
$$

Comparing Eqs. (14), one gets

$$
\mathbf{P}=\mathbf{V U}
$$

$$
\|\mathbf{P}\|=1 .
$$


Expanding VU into Twiss and coupling parameters defined in Edwards-Teng parametrization, one obtains

$$
\begin{gathered}
\mathbf{P}=\left(\begin{array}{ll}
\mathbf{P}_{11} & \mathbf{P}_{12} \\
\mathbf{P}_{21} & \mathbf{P}_{22}
\end{array}\right)=\left(\begin{array}{ccc}
r \mathbf{U}_{1} & \mathbf{C} \mathbf{U}_{2} \\
-\mathbf{C}^{+} \mathbf{U}_{1} & r \mathbf{U}_{2}
\end{array}\right), \\
\mathbf{P}=\left(\begin{array}{cccc}
r \sqrt{\beta_{I}} & 0 & c_{11} \sqrt{\beta_{I I}}-\frac{c_{12} \alpha_{I I}}{\sqrt{\beta_{I I}}} & -\frac{c_{12}}{\sqrt{\beta_{I I}}} \\
-\frac{\alpha_{I} r}{\sqrt{\beta_{I}}} & \frac{r}{\sqrt{\beta_{I}}} & c_{21} \sqrt{\beta_{I I}}-\frac{c_{22} \alpha_{I I}}{\sqrt{\beta_{I I}}} & \frac{c_{22}}{\sqrt{\beta_{I I}}} \\
-\frac{c_{12} \alpha_{I}}{\sqrt{\beta_{I}}}-c_{22} \sqrt{\beta_{I}} & \frac{c_{12}}{\sqrt{\beta_{I}}} & r \sqrt{\beta_{I I}} & 0 \\
\frac{c_{11} \alpha_{I}}{\sqrt{\beta_{I}}}+c_{21} \sqrt{\beta_{I}} & -\frac{c_{11}}{\sqrt{\beta_{I}}} & -\frac{\alpha_{I I} r}{\sqrt{\beta_{I I}}} & \frac{r}{\sqrt{\beta_{I I}}}
\end{array}\right) .
\end{gathered}
$$

Matrix $\mathbf{P}$ is independent of the Edwards-Teng parametrization. However, since Edwards-Teng parametrization gives the well-defined Twiss parameters and the coupling matrix $\mathbf{C}$, we would like to directly use them in the actionangle parametrization.

Twiss and coupling parameters can be calculated from matrix $\mathbf{P}$. The two eigenmodes' Twiss parameters and $r$ can be obtained by

$$
\begin{aligned}
& \left\{\begin{array}{l}
\beta_{I}=\frac{p_{11}}{p_{22}} \\
\alpha_{I}=-\frac{p_{21}}{p_{22}} \\
\gamma_{I}=\frac{1+\alpha_{I}^{2}}{\beta_{I}}=\frac{p_{21}^{2}+p_{22}^{2}}{p_{11} p_{22}}
\end{array},\right. \\
& \left\{\begin{array}{l}
\beta_{I I}=\frac{p_{33}}{p_{44}} \\
\alpha_{I I}=-\frac{p_{43}}{p_{44}} \\
\gamma_{I I}=\frac{1+\alpha_{I I}^{2}}{\beta_{I I}}=\frac{p_{43}^{2}+p_{44}^{2}}{p_{33} p_{44}}
\end{array}\right. \\
& r=\sqrt{p_{11} p_{22}}=\sqrt{p_{33} p_{44}} .
\end{aligned}
$$

The coupling matrix $\mathbf{C}$ can be obtained, for example, through $\mathbf{P}_{12}$ and $\mathbf{U}_{2}$,

$$
\mathbf{C}=\mathbf{P}_{12} \mathbf{U}_{2}^{-1}=\frac{\mathbf{P}_{12} \mathbf{P}_{22}^{-1}}{r} \text {. }
$$

For matrix $\mathbf{P}$, there are eight independent parameters since $\mu_{I}$ and $\mu_{I I}$ have been specified. There are different ways to define these eight independent parameters.

\section{Experimental construction of matrix $\mathbf{P}$}

To fully construct matrix $\mathbf{P}$, turn-by-turn $\left(x, x^{\prime}, y, y^{\prime}\right)$ data at one point are needed. In each interaction region (IR) of the Relativistic Heavy Ion Collider (RHIC), there are two DX/BPMs which are close to the last separation magnet DX and facing the interaction point (IP). There is no other magnet between the two BPMs if one ignores detector magnets. Turn-by-turn angles $x^{\prime}$ and $y^{\prime}$ at the two BPMs can be obtained through $x$ and $y$ readings at these two DX/ BPMs together with their separation distance.

The two DX/BPMs in IR8 in the Blue ring of RHIC are considered. The design distance from the two BPMs to the design IP is $8.33 \mathrm{~m}$. We designate the DX/BPM in the upstream of IP8 as g7-bx, and the DX/BPM in the downstream of IP8 as g8-bx. In the following the simulation code SAD [17] is used to simulate the turn-by-turn BPM data. In the simulation, only one free oscillation particle circulates in the Blue ring.

The uncoupled tunes are $\mu_{x, 0}=28.22, \mu_{y, 0}=29.23$. The skew quadrupole family 1 's integrated strength is set to be $0.0005 \mathrm{~m}^{-1}$ to introduce coupling into the uncoupled accelerator model. From a fast Fourier transform of $(x+y)$ turn-by-turn data at g7-bx, the two tunes are obtained $28.2126,29.2375$, respectively.

\section{Initial phases}

Here the initial phases of the two eigenmodes are calculated. Using harmonic analysis, $\phi_{I, 0}$ for eigenmode I at one point is given by

$$
\phi_{I, 0}=\arctan \left(\frac{-S_{I}}{C_{I}}\right)
$$

where

$$
\left\{\begin{array}{l}
C_{I}=\sum_{i=1}^{N} x_{i} \cos \left[2 \pi \mu_{I}(n-1)\right] \\
S_{I}=\sum_{i=1}^{N} x_{i} \sin \left[2 \pi \mu_{I}(n-1)\right]
\end{array} .\right.
$$

$N$ is the maximum data-taking turn.

The similar procedure is followed for $y$ turn-by-turn BPM data to get $\phi_{I I, 0}$ for eigenmode II,

$$
\phi_{I I, 0}=\arctan \left(\frac{-S_{I I}}{C_{I I}}\right)
$$

where

$$
\left\{\begin{array}{l}
C_{I I}=\sum_{i=1}^{N} y_{i} \cos \left[2 \pi \mu_{I I}(n-1)\right] \\
S_{I I}=\sum_{i=1}^{N} y_{i} \sin \left[2 \pi \mu_{I I}(n-1)\right]
\end{array} .\right.
$$

From turn-by-turn BPM tracking data at g7-bx, eigenmode I's and II's initial phases are $138.6449^{\circ}$ and $-152.1502^{\circ}$, respectively. At g8-bx, eigenmode I's and II's initial phases are $305.0668^{\circ}$ and $14.0882^{\circ}$, respectively, so the phase advances between the two BPMs are $166.4219^{\circ}$ and $166.2384^{\circ}$ for eigenmode I and II, respectively. From an analytical calculation with the simulation code SAD, they are $166.4212^{\circ}$ and $166.2399^{\circ}$, respectively. 


\section{Matrix F}

Equation (1) can be rewritten as

$$
\left(\begin{array}{c}
x \\
x^{\prime} \\
y \\
y^{\prime}
\end{array}\right)=\mathbf{F}\left(\begin{array}{c}
\cos \Phi_{I} \\
\sin \Phi_{I} \\
\cos \Phi_{I I} \\
\sin \Phi_{I I}
\end{array}\right)
$$

F can be obtained from turn-by-turn $\left(x, x^{\prime}, y, y^{\prime}\right)$ data. For example,

$$
\left\{\begin{array}{l}
F_{11}=\frac{2}{N} \sum_{i=1}^{N} x_{i} \cos \left[2 \pi \mu_{I}(n-1)+\phi_{I, 0}\right] \\
F_{12}=\frac{2}{N} \sum_{i=1}^{N} x_{i} \sin \left[2 \pi \mu_{I}(n-1)+\phi_{I, 0}\right] \\
F_{13}=\frac{2}{N} \sum_{i=1}^{N} x_{i} \cos \left[2 \pi \mu_{I I}(n-1)+\phi_{I I, 0}\right] \\
F_{14}=\frac{2}{N} \sum_{i=1}^{N} x_{i} \sin \left[2 \pi \mu_{I I}(n-1)+\phi_{I I, 0}\right]
\end{array}\right.
$$

Similarly from $x^{\prime}, y$, and $y^{\prime}$ turn-by-turn data, $F_{2 j}, F_{3 j}$, and $F_{4 j}, j=1,2,3,4$, can be obtained.

From the above tracking data at g7-bx, one obtains

$$
\mathbf{F}=\left(\begin{array}{cccc}
7.505 \times 10^{-4} & -1.852 \times 10^{-16} & -1.451 \times 10^{-4} & 5.040 \times 10^{-5} \\
-8.898 \times 10^{-5} & -1.061 \times 10^{-5} & 1.790 \times 10^{-5} & -3.912 \times 10^{-6} \\
4.579 \times 10^{-4} & 1.675 \times 10^{-4} & 2.390 \times 10^{-4} & 4.485 \times 10^{-17} \\
-5.187 \times 10^{-5} & -2.634 \times 10^{-5} & -2.831 \times 10^{-5} & -3.419 \times 10^{-6}
\end{array}\right)
$$

\section{Normalize matrix $\mathbf{F}$}

Matrix $\mathbf{F}$ includes action information. The ratio of the two actions is

$$
k=\sqrt{\frac{J_{I}}{J_{I I}}}=\sqrt{\frac{\left\|\mathbf{F}_{11}\right\|}{\left\|\mathbf{F}_{22}\right\|}}
$$

According to the definition of matrix $\mathbf{F}$, the signs of the elements in its second and fourth columns are inverted, and the first two column elements are divided by $k$. Normalizing the new matrix's determinant to be 1 , one gets matrix $\mathbf{P}$.

From simulated $\mathbf{F}$ in Eq. (44),

$$
\mathbf{P}=\left(\begin{array}{cccc}
6.873 & 1.696 \times 10^{-12} & -4.385 & -1.523 \\
-0.815 & 0.097 & 0.541 & 0.118 \\
4.193 & -1.534 & 7.225 & -1.356 \times 10^{-12} \\
-0.475 & 0.241 & -0.856 & 0.103
\end{array}\right)
$$

From matrix $\mathbf{P}$, Twiss and coupling parameters defined in Edwards-Teng parametrization can easily be calculated.

\section{Construct $\mathbf{P}$ from eigenvectors}

Here the procedure of $\mathbf{P}$ construction from the eigenvectors is tested. According to Eq. (14) and the measured eigentunes, the one-turn transfer map at $\mathrm{g} 7$-bx is obtained,

$$
\mathbf{T}=\left(\begin{array}{cccc}
8.403 & 69.305 & 0.194 & 1.039 \\
-0.988 & -8.028 & -0.015 & -0.055 \\
-0.198 & -2.212 & 8.339 & 69.348 \\
0.031 & 0.323 & -0.987 & -8.091
\end{array}\right)
$$

After finding the eigenvectors of matrix $\mathbf{T}$ and normalizing them according to the above given procedure to construct matrix $\mathbf{P}$ from eigenvectors, $\mathbf{P}$ at $\mathrm{g} 7$-bx is reconstructed,

$$
\mathbf{P}=\left(\begin{array}{cccc}
7.037 & 4.309 \times 10^{-16} & -4.285 & -1.488 \\
-0.834 & 0.099 & 0.529 & 0.116 \\
4.293 & -1.571 & 7.060 & 4.322 \times 10^{-16} \\
-0.486 & 0.247 & -0.836 & 0.101
\end{array}\right),
$$

which is very close to the simulated one in Eq. (46).

\section{PHASE ELLIPSES AND $\Sigma$ MATRIX}

\section{A. Projections of eigenmode I}

If only eigenmode I is considered, according to Eq. (6), the projections in $x-x^{\prime}, y-y^{\prime}, x-y$ planes are

$$
\begin{gathered}
\left(\begin{array}{c}
x \\
x^{\prime}
\end{array}\right)_{I}=\mathbf{P}_{11}\left(\begin{array}{c}
\sqrt{2 J_{I}} \cos \Phi_{I} \\
-\sqrt{2 J_{I}} \sin \Phi_{I}
\end{array}\right), \\
\left(\begin{array}{c}
y \\
y^{\prime}
\end{array}\right)_{I}=\mathbf{P}_{21}\left(\begin{array}{c}
\sqrt{2 J_{I}} \cos \Phi_{I} \\
-\sqrt{2 J_{I}} \sin \Phi_{I}
\end{array}\right), \\
\left(\begin{array}{l}
x \\
y
\end{array}\right)_{I}=\left(\begin{array}{cc}
p_{11} & 0 \\
p_{31} & p_{32}
\end{array}\right)\left(\begin{array}{c}
\sqrt{2 J_{I}} \cos \Phi_{I} \\
-\sqrt{2 J_{I}} \sin \Phi_{I}
\end{array}\right) .
\end{gathered}
$$


Normally these projections trace out different ellipses under the coupled situation. For example, the projection in the $x-x^{\prime}$ plane from eigenmode $\mathrm{I}$ is given by

$$
\left(p_{22} x\right)^{2}+\left(-p_{21} x+p_{11} x^{\prime}\right)^{2}=2 J_{I} r^{4},
$$

or in Twiss parameters,

$$
\gamma_{I} x^{2}+2 \alpha_{I} x x^{\prime}+\beta_{I}^{2} x^{\prime 2}=2 J_{I} r^{2} .
$$

These phase ellipses' areas are

$$
\left\{\begin{array}{l}
\epsilon_{I, x-x^{\prime}}=2 \pi J_{I} \cdot r^{2} \\
\epsilon_{I, y-y^{\prime}}=2 \pi J_{I} \cdot\left(1-r^{2}\right) \\
\epsilon_{I, x-y}=2 \pi J_{I} \cdot r\left|c_{12}\right|
\end{array} .\right.
$$

The phase area of eigenmode I is conserved,

$$
\epsilon_{I}=\epsilon_{I, x-x^{\prime}}+\epsilon_{I, y-y^{\prime}}=2 \pi J_{I} .
$$

The phase area partition ratio for eigenmode I is defined as

$$
\kappa_{I}=\frac{\epsilon_{I, y-y^{\prime}}}{\epsilon_{I, x-x^{\prime}}}=\frac{1-r^{2}}{r^{2}} .
$$

\section{B. Projections of eigenmode II}

Similarly, eigenmode II has projections in $y-y^{\prime}, x-$ $x^{\prime}, x-y$ planes,

$$
\begin{gathered}
\left(\begin{array}{c}
y \\
y^{\prime}
\end{array}\right)_{I I}=\mathbf{P}_{22}\left(\begin{array}{c}
\sqrt{2 J_{I I}} \cos \Phi_{I I} \\
-\sqrt{2 J_{I I}} \sin \Phi_{I I}
\end{array}\right), \\
\left(\begin{array}{c}
x \\
x^{\prime}
\end{array}\right)_{I I}=\mathbf{P}_{12}\left(\begin{array}{c}
\sqrt{2 J_{I I}} \cos \Phi_{I I} \\
-\sqrt{2 J_{I I}} \sin \Phi_{I I}
\end{array}\right), \\
\left(\begin{array}{c}
x \\
y
\end{array}\right)_{I I}=\left(\begin{array}{cc}
p_{13} & p_{14} \\
p_{33} & 0
\end{array}\right)\left(\begin{array}{c}
\sqrt{2 J_{I I}} \cos \Phi_{I I} \\
-\sqrt{2 J_{I I}} \sin \Phi_{I I}
\end{array}\right) .
\end{gathered}
$$

These phase ellipses' areas are

$$
\left\{\begin{array}{l}
\epsilon_{I I, y-y^{\prime}}=2 \pi J_{I I} \cdot r^{2} \\
\epsilon_{I I, x-x^{\prime}}=2 \pi J_{I I} \cdot\left(1-r^{2}\right) \\
\epsilon_{I I, x-y}=2 \pi J_{I I} \cdot r\left|c_{12}\right|
\end{array} .\right.
$$

The phase projection area of eigenmode II is also conserved,

$$
\epsilon_{I I}=\epsilon_{I I, y-y^{\prime}}+\epsilon_{I I, x-x^{\prime}}=2 \pi J_{I I} .
$$

The phase area partition ratio for eigenmode II is defined as

$$
\kappa_{I I}=\frac{\epsilon_{I I, x-x^{\prime}}}{\epsilon_{I I, y-y^{\prime}}}=\frac{1-r^{2}}{r^{2}},
$$

then

$$
\kappa_{I}=\kappa_{I I}
$$

\section{C. $\Sigma$ matrix and tilt angle}

The beam size matrix $\Sigma$ can be calculated through action-angle parametrization. It is defined as $[18,19]$

$$
\Sigma=\left(\begin{array}{cccc}
\left\langle x^{2}\right\rangle & \left\langle x x^{\prime}\right\rangle & \langle x y\rangle & \left\langle x y^{\prime}\right\rangle \\
\left\langle x^{\prime} x\right\rangle & \left\langle x^{\prime 2}\right\rangle & \left\langle x^{\prime} y\right\rangle & \left\langle x^{\prime} y^{\prime}\right\rangle \\
\langle y x\rangle & \left\langle y x^{\prime}\right\rangle & \left\langle y^{2}\right\rangle & \left\langle y y^{\prime}\right\rangle \\
\left\langle y^{\prime} x\right\rangle & \left\langle y^{\prime} x^{\prime}\right\rangle & \left\langle y^{\prime} y\right\rangle & \left\langle y^{\prime 2}\right\rangle
\end{array}\right) .
$$

The beam sizes in the $x-y$ plane are measurable. Using Eq. (5), it is easy to obtain

$$
\left\{\begin{array}{l}
\left\langle x^{2}\right\rangle=p_{11}^{2}\left\langle J_{I}\right\rangle+p_{13}^{2}\left\langle J_{I I}\right\rangle+p_{14}^{2}\left\langle J_{I I}\right\rangle \\
\left\langle y^{2}\right\rangle=p_{31}^{2}\left\langle J_{I}\right\rangle+p_{32}^{2}\left\langle J_{I}\right\rangle+p_{33}\left\langle J_{I I}\right\rangle \\
\langle x y\rangle=p_{11} p_{31}\left\langle J_{I}\right\rangle+p_{13} p_{33}\left\langle J_{I I}\right\rangle
\end{array} .\right.
$$

Normally the projection of the beam in the $x-y$ plane is not an ellipse. However, here we still define the coupling tilt angle for the projection as

$$
\tan \Psi=\frac{2\langle x y\rangle}{\langle x\rangle^{2}-\langle y\rangle^{2}},
$$

which is valid for $\langle x\rangle^{2}-\langle y\rangle^{2} \neq 0$.

\section{PROPAGATION OF MATRIX P}

From Eq. (13), matrix $\mathbf{P}_{2}$ at point $s_{2}$ can be obtained,

$$
\left\{\begin{array}{l}
\tilde{\mathbf{P}}_{11}=\left(\mathbf{T}_{11} \mathbf{P}_{11}+\mathbf{T}_{12} \mathbf{P}_{21}\right) \mathbf{R}^{-1}\left(\Delta \Phi_{I}\right) \\
\tilde{\mathbf{P}}_{21}=\left(\mathbf{T}_{21} \mathbf{P}_{11}+\mathbf{T}_{22} \mathbf{P}_{21}\right) \mathbf{R}^{-1}\left(\Delta \Phi_{I}\right) \\
\tilde{\mathbf{P}}_{12}=\left(\mathbf{T}_{11} \mathbf{P}_{12}+\mathbf{T}_{12} \mathbf{P}_{22}\right) \mathbf{R}^{-1}\left(\Delta \Phi_{I I}\right) \\
\tilde{\mathbf{P}}_{22}=\left(\mathbf{T}_{21} \mathbf{P}_{12}+\mathbf{T}_{22} \mathbf{P}_{22}\right) \mathbf{R}^{-1}\left(\Delta \Phi_{I I}\right)
\end{array} .\right.
$$

In the following the quantities and matrices at point $s_{2}$ except $\mathbf{P}_{2}$ have overhead tildes to distinguish these at point $s_{1}$. $\mathbf{T}_{i j}$ 's are block matrix elements of the section transfer matrix $\mathbf{T}_{1 \rightarrow 2}$.

\section{A. Phase advances}

When only eigenmode I is considered, from Eq. (10),

$$
\begin{gathered}
\left(\begin{array}{c}
\tilde{p}_{11} \cos \left(\Phi_{I}+\Delta \Phi_{I}\right) \\
\tilde{p}_{21} \cos \left(\Phi_{I}+\Delta \Phi_{I}\right)-\tilde{p}_{22} \sin \left(\Phi_{I}+\Delta \Phi_{I}\right) \\
\tilde{p}_{31} \cos \left(\Phi_{I}+\Delta \Phi_{I}\right)-\tilde{p}_{32} \sin \left(\Phi_{I}+\Delta \Phi_{I}\right) \\
\tilde{p}_{41} \cos \left(\Phi_{I}+\Delta \Phi_{I}\right)-\tilde{p}_{42} \sin \left(\Phi_{I}+\Delta \Phi_{I}\right)
\end{array}\right) \\
=\mathbf{T}_{1 \rightarrow 2}\left(\begin{array}{c}
p_{11} \cos \Phi_{I} \\
p_{21} \cos \Phi_{I}-p_{22} \sin \Phi_{I} \\
p_{31} \cos \Phi_{I}-p_{32} \sin \Phi_{I} \\
p_{41} \cos \Phi_{I}-p_{42} \sin \Phi_{I}
\end{array}\right) .
\end{gathered}
$$

Defining

$$
\mathbf{G}=\mathbf{T}_{1 \rightarrow 2} \mathbf{P}_{1},
$$

from the first row of Eq. (68), the phase advance between two points for eigenmode I is obtained, 


$$
\Delta \Phi_{I}=\arctan \frac{G_{12}}{G_{11}},
$$

and

$$
\tilde{p}_{11}=\sqrt{G_{11}^{2}+G_{12}^{2}}
$$

Similarly for eigenmode II, one gets

$$
\begin{aligned}
& \Delta \Phi_{I I}=\arctan \frac{G_{34}}{G_{33}}, \\
& \tilde{p}_{33}=\sqrt{G_{33}^{2}+G_{34}^{2}} .
\end{aligned}
$$

\section{B. $\widetilde{\mathbf{P}}_{i j}$ at point $s_{2}$}

Knowing the eigenmode phase advances, matrix $\mathbf{P}_{2}$ at point $s_{2}$ can be rewritten as

$$
\begin{aligned}
& \widetilde{\mathbf{P}}_{11}=\frac{1}{\tilde{p}_{11}}\left(\begin{array}{ll}
G_{11} & G_{12} \\
G_{21} & G_{22}
\end{array}\right)\left(\begin{array}{cc}
G_{11} & -G_{12} \\
G_{12} & G_{11}
\end{array}\right), \\
& \widetilde{\mathbf{P}}_{21}=\frac{1}{\tilde{p}_{11}}\left(\begin{array}{ll}
G_{31} & G_{32} \\
G_{41} & G_{42}
\end{array}\right)\left(\begin{array}{cc}
G_{11} & -G_{12} \\
G_{12} & G_{11}
\end{array}\right), \\
& \widetilde{\mathbf{P}}_{12}=\frac{1}{\tilde{p}_{33}}\left(\begin{array}{ll}
G_{13} & G_{14} \\
G_{23} & G_{24}
\end{array}\right)\left(\begin{array}{cc}
G_{33} & -G_{34} \\
G_{34} & G_{33}
\end{array}\right), \\
& \widetilde{\mathbf{P}}_{22}=\frac{1}{\tilde{p}_{33}}\left(\begin{array}{ll}
G_{33} & G_{34} \\
G_{43} & G_{44}
\end{array}\right)\left(\begin{array}{cc}
G_{33} & -G_{34} \\
G_{34} & G_{33}
\end{array}\right) .
\end{aligned}
$$

It is easy to check that $\tilde{p}_{12}=0, \tilde{p}_{34}=0$.

\section{Twiss and coupling parameters at point $s_{2}$}

Twiss parameters at point $s_{2}$ can be obtained from matrix $\mathbf{P}_{2}$,

$$
\begin{gathered}
\left\{\begin{array}{l}
\tilde{\beta}_{I}=\frac{G_{11}^{2}+G_{12}^{2}}{G_{11} G_{22}-G_{12} G_{21}} \\
\tilde{\alpha}_{I}=-\frac{G_{11} G_{21}+G_{12} G_{22}}{G_{11} G_{22}-G_{12} G_{21}}
\end{array},\right. \\
\left\{\begin{array}{l}
\tilde{\beta}_{I I}=\frac{G_{33}^{2}+G_{34}^{2}}{G_{33} G_{44}-G_{34} G_{43}} \\
\tilde{\alpha}_{I I}=-\frac{G_{33} G_{43}+G_{34} G_{44}}{G_{33} G_{44}-G_{34} G_{43}}
\end{array}\right.
\end{gathered}
$$

The coupling parameter $\tilde{r}$ at $s_{2}$ is given by

$$
\tilde{r}=\sqrt{\tilde{p}_{11} \tilde{p}_{22}}=\sqrt{G_{11} G_{22}-\bar{G}_{12} \bar{G}_{21}}
$$

or

$$
\tilde{r}=\sqrt{\tilde{p}_{33} \tilde{p}_{44}}=\sqrt{G_{33} G_{44}-G_{34} \bar{G}_{43}}
$$

From Eqs. (38) and (67), one gets

$$
\begin{aligned}
\tilde{r} \tilde{\mathbf{C}}= & \mathbf{T}_{11} \mathbf{T}_{21}^{-1}+\mathbf{T}_{12} \mathbf{T}_{22}^{-1}+\mathbf{T}_{11}(r \mathbf{C}) \mathbf{T}_{22}^{-1} \\
& +\mathbf{T}_{12}\left(r \mathbf{C}^{-1}\right) \mathbf{T}_{21}^{-1} .
\end{aligned}
$$

Under the uncoupled situation, it is easy to prove

$$
\left\{\begin{array}{l}
\Delta \Phi_{I}=\arctan \frac{p_{22} t_{12}}{p_{11} t_{11}+p_{21} t_{12}} \\
\Delta \Phi_{I I}=\arctan \frac{p_{44} t_{34}}{p_{33} t_{33}+p_{43} t_{34}}
\end{array},\right.
$$

or in Twiss parameters at $s_{1}$,

$$
\left\{\begin{array}{l}
\Delta \Phi_{I}=\arctan \frac{t_{12}}{\beta_{1} t_{11}-\alpha_{1} t_{12}} \\
\Delta \Phi_{I I}=\arctan \frac{t_{34}}{\beta_{2} t_{33}-\alpha_{2} t_{34}}
\end{array} .\right.
$$

In uncoupled sections, from Eq. (67), one gets

$$
\left\|\tilde{\mathbf{P}}_{11}\right\|=\left\|\mathbf{P}_{11}\right\|
$$

Since $\tilde{r}^{2}=\left\|\tilde{\mathbf{P}}_{11}\right\|$ and $r^{2}=\left\|\mathbf{P}_{11}\right\|$, so $r$ keeps constant in the uncoupled section. And the propagation of coupling matrix Eq. (82) reduces to

$$
\tilde{\mathbf{C}}=\mathbf{T}_{11} \mathbf{C} \mathbf{T}_{22}^{-1}
$$

\section{ACKNOWLEDGMENTS}

The author thanks S. Peggs and R. Talman (Cornell) for the stimulating discussions on coupling observables. The author thanks W. Fischer, S. Peggs, T. Satogata, T. Roser, D. Trbojevic, and F. Zimmerman (CERN) for help and encouragement during the writing of this article. This work is supported by U.S. DOE under Contract No. DEAC02-98CH10886.

[1] E. D. Courant and H. S. Snyder, Ann. Phys. (Paris) 3, 1 (1958).

[2] G. Ripken, DESY Internal Report No. R1-70/04, 1970.

[3] F. Willeke and G. Ripken, in Physics of Particle Accelerators, edited by Melvin Month and Margaret Dienes, AIP Conf. Proc. No. 184 (AIP, New York, 1989).

[4] H. Wiedemann, Particle Accelerator Physics II, Nonlinear and Higher-Order Beam Dynamics (Springer-Verlag, Berlin, 1995).

[5] V. Lebedev and S. Bogacz, "Betatron Motion with Coupling of Horizontal and Vertical Degrees of Freedom," http://www.jlab.org/ lebedev/AccPhys/ (unpublished).

[6] L. C. Teng, NAL Report No. FN-229, 1971.

[7] D. Edwards and L. Teng, IEEE Trans. Nucl. Sci. 20, 3 (1973).

[8] S. Peggs, IEEE Trans. Nucl. Sci. 30, 2460 (1983).

[9] M. Billing, Cornell Report No. CBN 85-2, 1985.

[10] D. Sagan and D. Rubin, Phys. Rev. ST Accel. Beams 2, 074001 (1999). 
[11] L. Schachinger and R. Talman, Manual for the Program TEAPOT, Noninteractive Fortran Version, Appendix G, 1996 (unpublished).

[12] P. Bagley and D. Rubin, in Proceedings of the 1989 Particle Accelerator Conference, Chicago, IL (IEEE, Piscataway, NJ, 1989), p. 874.

[13] J. Irwin et al., Phys. Rev. Lett. 82, 1684 (1999).

[14] C.-x. Wang et al., Phys. Rev. ST Accel. Beams 6, 104001 (2003).
[15] D. Sagan, Phys. Rev. ST Accel. Beams 4, 122801 (2001).

[16] D. Sagan et al., Phys. Rev. ST Accel. Beams 3, 102801 (2000).

[17] SAD code homepage, http://acc-physics.kek.jp/SAD/ sad.html.

[18] K. Brown, CERN Yellow Report No. 80-04, 1980.

[19] F.C. Iselin, The MAD Program Version 8.13-Physicist Manual (unpublished), p. 49. 